$17 \mid 1998$

Geistige Eigentumsrechte, was steht für die Entwicklungsländer auf dem spiel?

\title{
7. Kultur und Wissenschaft
}

\section{(2) OpenEdition \\ Journals}

Electronic version

URL: http://journals.openedition.org/sjep/711

ISSN: 1663-9677

\section{Publisher}

Institut de hautes études internationales et du développement

\section{Printed version}

Date of publication: 1 avril 1998

Number of pages: 265-274

ISSN: 1660-5926

\section{Electronic reference}

«7. Kultur und Wissenschaft », Schweizerisches Jahrbuch für Entwicklungspolitik [Online], 17 | 1998,

Online erschienen am: 03 August 2012, abgerufen am 07 September 2020. URL : http://

journals.openedition.org/sjep/711 


\section{KULTUR UND WISSENSCHAFT}

\subsection{ERZIEHUNG ZUR ENTWICKLUNG}

Der Globalisierungsprozess, der in den letzten Jahren auf zahlreichen Gebieten wie Handel, Finanzen, Kommunikation, Kultur und Politik eingesetzt hat, sowie die Folgen der Umweltzerstörung und der Migrationsbewegungen bewirkten für die einzelnen Menschen einen weitreichenden Verlust an Stabilität. Gründe für diese Destabilisierung sind die Verdrängung althergebrachter Modelle durch die wachsende kulturelle Homogenisierung, die zunehmend komplexen internationalen Beziehungen und die weltweit spürbaren Auswirkungen der Armuts- und Umweltproblematik, die die Durchführung von Gegenmassnahmen erschweren. Eltern, Unterrichtende und Erzieher müssen nicht nur mit ihren eigenen Ungewissheiten fertig werden, sondern sie sehen sich zudem mit den Hinterfragungen einer Jugend konfrontiert, deren Bild von der Welt und der Zukunft durch Skepsis geprägt ist, und die wenigstens Ansätze von Antworten und Hilfestellungen verlangt.

Die Globalisierung hat aber auch, besonders bei der Direktion für Entwicklung und Zusammenarbeit (DEZA) und bei Nichtregierungsorganisationen, das Bewusstsein dafür gefördert, dass die zunehmenden internationalen Verflechtungen nach einem neuen pädagogischen Engagement verlangen, um die Jugend auf die Welt von morgen vorzubereiten. Seit 1970 stellt die DEZA pädagogische Hilfsmittel zur Verfügung, die das Verständnis für die Ursachen der Unterentwicklung fördern sollen. Private Organisationen wie etwa die Arbeitsgemeinschaft der Hilfswerke, die 1975 die Schulstelle Dritte Welt in Bern, 1981 den Service école Tiers Monde in Lausanne und 1987 das Centro Terzo Mondo in Lugano gründete, oder das Schweizerische Komitee für UNICEF und insbesondere die Erklärung von Bern, haben die Frage nach den Einflussmöglichkeiten des einzelnen Menschen ins Zentrum ihrer pädagogischen Arbeit gestellt. In dem 1982 ins Leben gerufenen Forum «Schule für eine Welt» sind die meisten Organisationen vertreten, die sich für die Förderung des Verständnisses der Jugend in den Bereichen Nord-Süd-Beziehungen, Menschenrechte, Umwelt, Bekämpfung des Rassismus und für die interkulturelle Erziehung einsetzen.

1987 haben die Mitgliedsorganisationen des Forums «Schule für eine Welt» das Konzept Erziehung zur Entwicklung folgendermassen zu definieren versucht: «Förderung der persönlichen Entfaltung der Kinder und Jugendlichen in ihrer Gemeinschaft hier und weltweit. In dieser Entfaltung begreifen sich die Heranwachsenden immer mehr als Teil einer sich wandelnden Welt, in der alle aufeinander angewiesen sind. Die Erziehung zur Entwicklung ermutigt uns, an der Gestaltung der Zukunft aktiv teilzunehmen und die Grundwerte der Würde, der Gerechtigkeit und der Solidarität für eine weltweite Gemeinschaft zu verwirklichen ${ }^{\prime}$.

1. Bericht der Pädagogischen Kommission des Forums «Schule für eine Welt », Globales Lernen - Anstösse für die Bildung in einer vernetzten Welt, September 1996, S. 11. 


\section{$\square$ Gründung der Stiftung "Bildung und Entwicklung 》}

Im Februar 1997 wurde in der Schweiz im Bereich der Förderung der Erziehung zur Entwicklung ein wichtiger Schritt getan. Die Konferenz der kantonalen Erziehungsdirektoren (EDK), die Direktion für Entwicklung und Zusammenarbeit (DEZA), die im Dachverband «Schule für eine Welt» zusammengeschlossenen Nichtregierungsorganisationen, die Lehrerinnen- und Lehrerverbände sowie andere Beteiligte gründeten gemeinsam die Stiftung «Bildung und Entwicklung». Anlässlich der Pressekonferenz betonte Walter Fust, Direktor der DEZA : «Während wir, die DEZA, bereits seit bald 20 Jahren die Bildungsarbeit der privaten Hilfswerke finanziell unterstützen, ist die Intensivierung der Zusammenarbeit mit den Lehrerinnen- und Lehrerverbänden sowie der für die schulische Bildung primär zuständigen Kantone ein wichtiger und unerlässlicher Schritt in Richtung einer breiteren Abstützung der auf eine globale Sichtweise verpflichteten Bildung unserer Jugend. »

Die Gründung dieser Stiftung ist das Ergebnis langjähriger Bemühungen. 1993 haben die Mitglieder des Forums «Schule für eine Welt» damit begonnnen, das Angebot der NRO (die von ihnen geleistete Arbeit im Bereich der Erziehung zur Entwicklung, die Bereitstellung pädagogischer Hilfsmittel, Aktionsvorschläge usw.) den Bedürfnissen der Unterrichtenden gegenüberzustellen. Aus den Ergebnissen dieser Studie, die unter dem Titel «L'apprentissage global en Suisse Analyse de la situation, des attentes et des perspectives ${ }^{2}$ veröffentlicht wurden, ging klar hervor, dass die Arbeit der NRO besser koordiniert werden muss, um den Unterrichtenden die Einflechtung von Themen aus dem Bereich Erziehung zur Entwicklung in ihren Unterricht zu erleichtern. Zudem verlangt die Agenda 21, der Aktionsplan des Erdgipfels in Rio, seit 1992 von allen Ländern besondere Anstrengungen zur Verstärkung der Bildung in Umwelt- und Entwicklungsfragen.

Die Errichtung der Stiftung wurde im Laufe des Jahres 1997 kontinuierlich vorangetrieben. Die Tätigkeiten der Schulstellen der Arbeitsgemeinschaft der Hilfswerke und des Forums «Schule für eine Welt» wurden sukzessive der neuen Stiftung übertragen, danach wurden diese Organe aufgelöst. Die Stiftung «Bildung und Entwicklung» versteht sich in erster Linie als Ansprechpartner für Unterrichtende, pädagogische Dokumentationszentren, Verantwortliche für die Lehrerfortbildung und für Lehrplan- und Lehrmittelkommissionen. Sie klärt die Bedürfnisse der Unterrichtenden ab, evaluiert Unterrichtsmaterialien, unterstützt deren Verbreitung, fördert die Innovation in Schulen und Lehrplänen und wirkt bei der Aus- und Weiterbildung der Unterrichtenden mit.

Ziel der Stiftung ist die Unterstützung einer Ausbildung, die Wissen vermittelt und gleichzeitig das Verständnis für globale Zusammenhänge entwickelt, die konkrete Möglichkeiten zur Einflussnahme aufzeigt und die Fähigkeiten des einzelnen fördert, Beziehungen über die sprachlichen, kulturellen und geographischen Grenzen hinaus aufzubauen. Mittelfristig wird die Stiftung Bildung und Entwicklung pädagogische Hilfsmittel und Bildungsprojekte für Schulen finanziell unterstützen. In einer zweiten Phase soll auch die ausserschulische Bildung gefördert werden.

2. Forum «Schule für eine Welt», L'apprentissage global en Suisse - Analyse de la situation, des attentes et des perspectives, 1995. 
Die Stiftung erbringt ihre Dienstleistungen von vier Standorten aus. Die Zentralstelle in Bern ${ }^{3}$ soll Aufgaben auf nationaler und internationaler Ebene wahrnehmen, während die drei Regionalstellen (Zürich, Lausanne und Lugano) für Beratungs- und Unterstützungsdienste für Schulen und Unterrichtende aller Stufen, Evaluierung von Unterrichtsmaterialien sowie Aus- und Weiterbildung zuständig sind.

\section{$\square$ Studie über das Weltbild von Jugendlichen}

Auf Initiative des Forums «Schule für eine Welt» und der DEZA führte das Institut für Pädagogik der Universität Bern eine umfassende Studie über das Weltbild von Jugendlichen durch. Der Schlussbericht mit dem Titel «Die Schweiz, die Entwicklungsländer und globale Zusammenhänge aus der Sicht von Jugendlichen» wurde im Juni 1997 veröffentlicht ${ }^{4}$. Die Studie ist die Weiterführung einer 1985 durchgeführten Untersuchung ${ }^{5}$ und gibt Aufschluss über die in den letzten 10 Jahren stattgefundenen Veränderungen im Weltbild und in der Einstellung von Jugendlichen.

Die Untersuchung umfasst vier Themenschwerpunkte: Das Zusammenleben von Menschen verschiedener Nationalitäten in der Schweiz, die Schweiz aus der Sicht der Jugendlichen, das Bild, das sich die Jugendlichen von den Entwicklungsländern machen, und die weltweiten Verflechtungen aus der Sicht der Jugendlichen. Der Fragebogen bezog sich auf die Kenntnisse der Jugendlichen, auf ihre Einstellung und ihr Verhalten und auf ihre Handlungsbereitschaft in vier genannten Bereichen. Ausgehend von einer repräsentativen Auswahl haben knapp 5000 Schüler im Alter von 13 bis 16 Jahren aus allen drei Sprachregionen an der Erhebung teilgenommen. Ihnen wurde ein umfangreicher Fragebogen mit 88 Fragen vorgelegt. Im folgenden werden die wichtigsten Elemente der Antworten vorgestellt. ${ }^{6}$

Bild der Schweiz

Um zu ermitteln, welches Bild sich die Jugendlichen von der Schweiz machen, wurden ihnen bestimmte Problemstellungen vorgelegt. Insgesamt wurden die Drogen als grösstes Problem bezeichnet, gefolgt von Arbeitslosigkeit und AIDS. Aber auch die Gewalt, die Umweltzerstörung und der Rassismus wurden als Problembereiche identifiziert. Die Flüchtlingsproblematik, die Europadiskussion, die Nuklearenergie, die Armut und die Überbevölkerung wurden ebenfalls noch als wichtige Problemgruppen genannt. Hingegen wurden die politische Situation, die Unterernährung, die Bildung und der Zugang zu medizinischer Pflege als weniger gravierende oder gar nicht existierende Probleme bezeichnet ${ }^{7}$. Beim

3. Stiftung Bildung und Entwicklung, Monbijoustrasse 31, Postfach 8366, 3001 Bern, Tel. 031/382 80 80, Fax 031/382 8082 .

4. Schlussbericht «Das Weltbild von Jugendlichen », Institut für Pädagogik der Universität Bern in Zusammenarbeit mit dem Forum «Schule für eine Welt», Bern, Juni 1997. Nur auf deutsch erhältlich, ca. 500 Seiten. Eine Kurzfassung der Studie ist auf Deutsch und Französisch erhältlich: W. Herzog, J. Guldimann, T. Oegerli, Die Schweiz, die Entwicklungsländer und globale Zusammenhänge aus der Sicht von Jugendlichen, eine gesamtschweizerische Erhebung bei 13- bis 16jährigen, Institut für Pädagogik der Universität Bern und Forum «Schule für eine Welt», Bern, Juni 1997.

5. Monique Hirsch-Cahannes und Ueli Tecklenburg: Die Welt, in der wir leben. Lausanne: 1985.

6. Weitere Fragen zu den Themen Entwicklungszusammenarbeit, Freundschaft zwischen Schweizern und Ausländern, Motive, die das Asyl rechtfertigen, Einsatzbereitschaft der Jugendlichen zugunsten der Entwicklungsländer usw. werden im Schlussbericht ausführlich diskutiert.

7. Bei den hier vorgestellten Resultate handelt es sich um globale Ergebnisse. Bei einer detaillierteren Analyse dürften je nach Sprachregion, Geschlecht oder Alter der Jugendlichen durchaus Unterschiede erkennbar sein. Weitere Details sind der vollständigen Studie zu entnehmen. 
Vergleich mit der Studie aus dem Jahr 1985 fällt auf, dass vor etwa zehn Jahren die Sorge um die Umwelt an erster Stelle stand, während sie in dieser neuen Studie «nur» den fünften Platz einnimmt.

\section{Bild der Entwicklungsländer}

Das Bild, das sich die Jugendlichen von den Entwicklungsländern machen, ist vorwiegend negativ besetzt. Für sie ist die Realität in diesen Ländern in erster Linie von Armut, Hunger, Überbevölkerung und Krankheit bestimmt. In den vergangenen zehn Jahren hat sich das Bild der Jugendlichen von den Entwicklungsländern kaum verändert. Ihrer Ansicht nach stellen die Unterernährung, die Armut, die Arbeitslosigkeit, der Krieg und die ungenügende medizinische Versorgung die wichtigsten Probleme dieser Länder dar.

\section{Beziehungen zwischen der Schweiz und den Entwicklungsländern}

Die Lage der Entwicklungsländer wird nach Ansicht der Jugendlichen hauptsächlich durch das Verhalten der grossen multinationalen Unternehmen, das ungünstige Klima, die durch Ungerechtigkeit gekennzeichneten sozialen Strukturen, das hohe Bevölkerungswachstum und die hohen Zinssätze der Banken (Schuldenproblematik) bestimmt. Die Schüler sind der Meinung, dass die Schwierigkeiten der Entwicklungsländer weder auf «den Verkauf von Produkten schlechter Qualität» noch auf «Faulheit» zurückgeführt werden können.

Zusammenleben von Menschen verschiedener Nationalitäten in der Schweiz Die Haltung der Jugendlichen gegenüber Ausländern wurde eruiert, indem ihnen drei mögliche Szenarien des Zusammenlebens präsentiert wurden. Diese Szenarien waren die Toleranz (Ausländer sollen in der Schweiz so leben können, wie es den Lebensgewohnheiten in ihrem Heimatland entspricht), die Abgrenzung (Ausländer sollen in der Schweiz so leben, dass ihre Lebensgewohnheiten die Lebensgewohnheiten der Schweizer nicht stören) und die Anpassung (Ausländer sollen in der Schweiz ihre Lebensgewohnheiten den Lebensgewohnheiten der Schweizer anpassen). Die eine Hälfte der Schüler sprach sich zugunsten der zweiten Variante aus $(48,9 \%)$, die anderen entschieden sich eher für die Anpassung $(28,8 \%)$ als für ein von Toleranz geprägtes Zusammenleben $(22,3 \%)$.

\section{Informationsquellen}

In der Studie wurde ebenfalls untersucht, woher die Jugendlichen ihre Informationen über die Entwicklungsländer beziehen. Das Fernsehen wurde von $85 \%$ der Befragten als wichtigste Informationsquelle bezeichnet, gefolgt von der Schule $(68 \%)$ und den Zeitungen $(66 \%)$. Die Familie scheint in dieser Hinsicht eine weniger wichtige Rolle zu spielen, aber immerhin wird sie noch von gut der Hälfte $(53 \%)$ der Schüler genannt. Der Vergleich mit der Umfrage aus dem Jahre 1985 ergibt, dass die Schule vom dritten auf den zweiten Rang aufgestiegen ist: 1996 wurde sie eineinhalb mal öfter als Informationsquelle genannt als 1985.

Das Bild, das sich die Jugendlichen von den Entwicklungsländern machen, hat sich in den vergangenen zehn Jahren kaum verändert. Es handelt sich dabei um ein Stereotyp, das weitgehend den durch das Fernsehen verbreiteten Informationen entspricht. Immerhin konnte sich die Schule als Informationsquelle auf den zweiten Rang verbessern. Insgesamt dürften die Ergebnisse dieser Studie in erster Linie bei der Ausarbeitung neuer pädagogischer Hilfsmittel von Nutzen sein. 
QUELLEN

B. Riondet, Education au développement, Hachette éducation, Paris : 1995.

Pressedossier vom 18. Februar 1997, Gründung der Stiftung «Bildung und Entwicklung », erhältlich beim Pressedienst der DEZA, Tel. 031/322 3488 .

Bericht der Pädagogischen Kommission des Forums «Schule für eine Welt », Globales Lernen - Anstösse für die Bildung in einer vernetzten Welt, September 1997.

W. Herzog, J. Guldimann, T. Oegerli, Die Schweiz, die Entwicklungsländer und globale Zusammenhänge aus der Sicht von Jugendlichen, Eine gesamtschweizerische Erhebung bei 13-bis 16jährigen Jugendlichen zur Schweiz, zu den Entwicklungsländern und zu globalen Zusammenhängen. Kurzfassung der Studie unter dem Titel «Das Weltbild von Jugendlichen », herausgegeben vom Institut für Pädagogik der Universität Bern und dem Forum «Schule für eine Welt», Bern, Juni 1997, 60 Seiten.

Mosquito, «Neue Strukturen für Bildung und Entwicklung », Nr. 7/97.

\subsection{FORSCHUNGS- UND WISSENSCHAFTSZUSAMMENARBEIT}

\section{$\square$ Die Schweiz im globalen Wandel. Schlussfolgerungen des NFP 28.}

Das Nationale Forschungsprogramm 28 (NFP 28) «Aussenwirtschaftliche und entwicklungspolitische Herausforderungen » wurde im September mit der Veröffentlichung des Schlussberichts von Rolf Kappel und Olivier Landmann abgeschlossen ${ }^{8}$. In den 26 Forschungsprojekten, die von 1989 bis 1996 durchgeführt wurden, ging es um Themen aus den Bereichen Aussenwirtschaft und Entwicklungspolitik. Der Schlussbericht wurde anlässlich eines öffentlichen Seminars in Bern am 25. September 1997 vorgestellt. Verschiedene Synthesen befassen sich mit der schweizerischen Aussenpolitik in bezug auf die Entwicklungsländer (siehe Kasten). Die folgenden Schlussfolgerungen und Vorschläge gehören zu den Hauptergebnissen der Projekte im Zusammenhang mit der schweizerischen Politik gegenüber den Entwicklungsländern.

- Für die Probleme der schweizerischen Wirtschaft ist nicht die Globalisierung verantwortlich. Die Schwierigkeiten des Wirtschaftsplatzes Schweiz gehen nicht auf die Produktionsauslagerung, sondern auf den überbeschützten nationalen Sektor zurück. Zwischen dem im Ausland wettbewerbsfähigen internationalen Sektor (Konkurrenzdruck) und den internen Überkapazitäten (z.B. im Bankwesen) besteht ein deutliches Gefälle.

口 Die schweizerische Gastarbeiterpolitik bremst das Wirtschaftswachstum. Die Einwanderungsregelungen wurden umgangen und dazu eingesetzt, um strukturell schwache, ausschliesslich binnenwirtschaftlich orientierte Wirtschaftszweige (z.B. Bauwirtschaft) zu unterstützen. Thomas Straubhaar, der Autor eines der Forschungsprojekte, schlägt eine Migrationspolitik vor, die vermehrt auf den präzisen Bedarf des Schweizer Markts an qualifizierten Arbeitskräften ausgerichtet werden soll. Nach diesem innovativen Vorschlag hätten grundsätzlich alle Weltbürger/innen ein Anrecht, in die Schweiz einzuwandern, sofern sie eine Einwanderungsabgabe entrichten. Diese Gebühr sollte die Schweiz für die abgetretenen öffentlichen Leistungen, Einrichtungen und Rechte entschädigen und die Integrations- und Assimilierungskosten decken. Andererseits sollte die schweizerische Aussenpolitik zum Abbau der Migrationsursachen beitragen, damit potentielle Einwanderer in ihrem Herkunftsland in Verhältnissen leben können, die sie zum Bleiben bestimmen.

8. Kappel, Rolf; Landmann, Olivier; Die Schweiz im globalen Wandel. Aussenwirtschaftliche und entwicklungspolitische Herausforderungen, Zürich, Verlag Neue Zürcher Zeitung, 1997, 456 S. 
๑ Die Schweizer Wirtschaft (und die Diplomatie) haben ihre Chancen auf den dynamischsten asiatischen Märkten noch nicht ausreichend genutzt.

- Die Schweiz steht vor den gleichen Herausforderungen wie die Entwicklungsländer: Öffnung ihrer Volkswirtschaft nach aussen. Isolierung und Behinderung der Strukturanpassung verursachen erhebliche volkswirtschaftliche Kosten.

口 Laut den Autoren einiger Forschungsprojekte des NFP 28 gibt es immer noch keine überzeugende Alternative zu den in den Entwicklungsländern durchgeführten Strukturanpassungsprogrammen. Oft werden die nachteiligen Folgen der Programme für die ärmsten Bevölkerungsschichten kritisiert, vor allem die Auswirkungen der Senkung der öffentlichen Sozialausgaben (Ausbildung, Gesundheit). Gemäss den Studien ist es allerdings schwierig, die negativen sozialen Auswirkungen des Anpassungsprozesses auf die ärmsten Bevölkerungsschichten zu evaluieren (die Konsequenzen der Strukturanpassung und die Folgen einer wirtschaftlichen Krisensituation lassen sich nicht leicht auseinanderhalten). Oft berücksichtigen die Kritiken nicht, wie sich die soziale Lage ohne Anpassungen entwickelt hätte. Mit der folgenden Aussage gehen die Autoren noch einen Schritt weiter. Nach ihrer Auffassung kann man von Strukturanpassungsprogrammen nicht verlangen, mehrere Probleme gleichzeitig zu lösen: wirtschaftliche Stabilisierung eines Landes und Öffnung nach aussen, Bekämpfung der Armut und gleichzeitig Förderung der nachhaltigen Entwicklung. Diese drei Ziele lassen sich nicht auf einmal mit nur einem Massnahmenkatalog erreichen.

\section{PROJEKTE DES NFP 28 ÜBER DIE ENTWICKLUNGSPOLITIK}

- Gilbert Etienne/Jean-Luc Maurer/Philippe Régnier/Christine Renaudin.

Suisse-Asie: Pour un nouveau partenariat Inde - Pakistan - Chine - Indonésie.

Bisher hat die Schweizer Privatwirtschaft gut auf die Entwicklung der vier asiatischen Länder reagiert. Allerdings könnte das Engagement der öffentlichen Hand und der Unternehmen ausgebaut werden (Handelsdiplomatie, Ausbildung von Kadern usw.)

- Jakob Juchler, Krise und Umbruch in Osteuropa.

Gesamthafte Betrachtungsweise des Transformationsprozesses in Osteuropa. Als bester Nährboden für Demokratie und Marktwirtschaft empfiehlt der Autor die Öffnung der Grenzen für osteuropäische Produkte.

口 Thomas Straubhaar. Von der Ausländer-zur Migrationspolitik.

Der Autor plädiert für eine neue Migrationspolitik: klare Regeln zur Einwanderung festlegen (Migrations-Innenpolitik) und Migrationsursachen abbauen (Migrations-Aussenpolitik).

口 Joseph Hunkeler. L'impossibilité de rembourser la dette.

Untersuchung des Strukturanpassungsprogramms in Burundi. Die überstürzte Liberalisierung des Aussenhandels und die schnelle interne Entschuldung des Staates führten zu einer untragbaren Aussenverschuldung.

- Jean-Max Baumer. Le programme d'ajustement structurel en Bolivie.

Analyse der Konsequenzen des Anpassungsprogramms. Die gesamtwirtschaftliche Stabilisierung war zwar ein Erfolg, konnte aber die Armut des Landes nicht beseitigen.

- Christian Suter: Weltwirtschaftliche Verflechtung, Regimewandel und Demokratisierung in Lateinamerika.

Der Fall Costa Rica zeigt, dass eine wirtschaftlich erfolgreiche Strukturanpassung in einem demokratischen Umfeld möglich und dauerhaft ist. 
- Gilbert Blardone/Lothar Caviezel. Stratégies de développement et ajustements structurels: Etude de l'appui de la Suisse au programme d'ajustement de deux de ses partenaires africains.

Analyse der wirtschaftlichen und sozialen Folgen der Strukturanpassungsprogramme in Madagaskar und Tansania.

- Martin Hellwig. Die internationale Schuldenproblematik: Ursachen, Entwicklungstendenzen, Lösungsmöglichkeiten aus der Sicht der Schweiz.

口 Othmart Schwank/Dieter Zürcher. Stadt-Land-Beziehungen: Eine Herausforderung für die schweizerische Entwicklungszusammenarbeit.

Am Beispiel Nepal werden fünf Aspekte der Stadt-Land-Beziehungen festgehalten, die für den Entwicklungsprozess besonders bedeutsam sind: Abwanderung aus ländlichen Gebieten, mangelnde Dezentralisierung von Entscheidungen, Ineffizienz staatlicher Institutionen im ländlichen Raum, mangelnde Innovationsfähigkeit und Externalisierung der Folgekosten der Verstädterung.

- Jean-Pierre Jacob/François Margot/Paul Sauvain/Peter Uvin. La contribution des acteurs publics et privés au développement rural local des pays du tiers-monde (Sénégal, Burkina Faso, Burundi, Bolivie). Rolle der lokalen Regierungs- und Verwaltungsstellen im Entwicklungsprozess.

口 Markus Wyss/Bruno Messerli/Thomas Straubhaar. Förderung einer ökologisch verträglichen wirtschaftlichen Zusammenarbeit mit weniger entwickelten Ländern. Und: Ernst Brugger/Martin Maurer. Fördern multinationale Firmen das Ökologie-Know-how in Entwicklungsländern?

Eine weniger restriktive Umweltgesetzgebung in den Entwicklungsländern bilden keine genügende Motivation, dass multinationale Unternehmen Teile der Produktion zu Töchtern in Drittweltländern auslagern.

- Richard Gerster: In gemeinsame Interessen investieren.

Das Forschungsprojekt untersucht die notwendige Reform der Entwicklungszusammenarbeit. Der Autor fordert vor allem den Abschluss langfristiger und symmetrischer Zusammenarbeitsverträge zwischen der Schweiz und den Schwerpunktländern der Entwicklungszusammenarbeit.

Quelle: Kastentext zitiert aus: Kappel, Rolf; Landmann, Olivier, Die Schweiz im globalen Wandel. Aussenwirtschaftliche und entwicklungspolitische Herausforderungen, Zürich, Verlag Neue Zürcher Zeitung, 1997, 456 S. Die Seiten 437 bis 453 des Werkes enthalten die Projekte des NFP 28 sowie die Liste der Veröffentlichungen.

\section{QUELLEN}

Kappel, Rolf; Landmann, Olivier, Die Schweiz im globalen Wandel. Aussenwirtschaftliche und entwicklungspolitische Herausforderungen. Zürich, Verlag Neue Zürcher Zeitung, 1997, 456 S.

Presseunterlagen des Schweizerischen Nationalfonds zur Förderung der wissenschaftlichen Forschung, Bern, 26. August 1997.

Neue Zürcher Zeitung, 26./27. September 1997 «Mehr Symmetrie zwischen Nord und Süd. Vom Forschungsprogramm zur Strukturanpassung».

\subsection{KULTURAUSTAUSCH SÜD-NORD}

\section{$\square$ Filme aus Entwicklungsländern in der Schweiz}

Der schweizerische Filmmarkt wird von gewissen Ländern klar dominiert, und die Stellung der Filme aus Entwicklungsländern ist entsprechend gering. Tabelle 18 gibt Aufschluss über die Anzahl Kinobesucher pro Herkunftsland der Filme. Die Statistik beruht auf Daten, die von der Mehrheit der kommerziellen Kinos in der Schweiz bekanntgegeben wurden. Die Besucherdaten von Filmfestivals und Filmklubs sind allerdings nicht berücksichtigt. Insgesamt lockten die rund 1400 Filme, die 1996 in den Schweizer Kinos vorgeführt wurden, mehr als 16,8 Millionen Zuschauer an. Der Marktanteil amerikanischer Produktionen verzeichnete 
in den letzten Jahren einen kontinuierlichen Rückgang: Lag er Anfang der 90er Jahre noch bei über $80 \%$, so verringerte er sich bis 1994 auf $75 \%$ und lag 1995 und 1996 nur mehr bei zwei Dritteln. Hingegen war beim Marktanteil von Filmen europäischer Provenienz in den vergangenen Jahren eine Verbesserung festzustellen, wobei der Löwenanteil 1996 auf französische und britische Produktionen entfiel. Obgleich gewisse Entwicklungsländer eine äusserst aktive Filmindustrie besitzen, ist der Anteil der Filme aus diesen Ländern sehr gering. 1996 entfielen lediglich 1,1\% aller Kinobesuche auf diese Produktionen.

Tabelle Nr. 18

Anzahl Kinobesuche nach Herkunftsland der Filme (1996)

\begin{tabular}{|c|c|c|c|}
\hline $\begin{array}{l}\text { Herkunftsland der } \\
\text { Filme }\end{array}$ & Besucherzahl & $\begin{array}{c}\text { in \% } \\
\text { der Gesamtbesucherzahl }\end{array}$ & $\begin{array}{r}\text { Anzahl der } \\
\text { Filme }\end{array}$ \\
\hline USA & $10^{\prime} 534^{\prime} 245$ & 62,6 & 571 \\
\hline Frankreich & 1'887'261 & 11,2 & 217 \\
\hline Grossbritannien & $982^{\prime} 715$ & 5,8 & 95 \\
\hline Italien & 960’306 & 5,7 & 46 \\
\hline Schweiz & 707’081 & 4,2 & 153 \\
\hline Deutschland & $570 ’ 130$ & 3,4 & 117 \\
\hline Zentral- und Osteuropa & $94^{\prime} 670$ & 0,6 & 31 \\
\hline Andere Industrieländer & 902’278 & 5,4 & 107 \\
\hline Entwicklungsländer & $189^{\prime} 438$ & 1,1 & 62 \\
\hline davon: Lateinamerika & $110^{\prime} 415$ & 0,7 & 13 \\
\hline Asien & 41’099 & 0,2 & 29 \\
\hline Afrika & $24^{\prime} 625$ & 0,1 & 13 \\
\hline Gesamtzahl Besucher/Filme & $16^{\prime} 8288^{\prime} 124$ & 100,0 & 1,399 \\
\hline
\end{tabular}

Die grosse Mehrheit der Kinobesucher sieht sich nur eine sehr beschränkte Anzahl Filme an. Allein rund ein Viertel aller Kinobesuche entfiel 1996 auf die zehn erfolgreichsten Filme. Unter den 100 «Bestsellern» des Jahres sind nur zwei Produktionen aus Entwicklungsländern zu finden: «Guantanamera»von Thomas Gutierrez Alea (Kuba, 71. Rang mit über 55'000 Besuchen) und « No te mueras sin decirme adonde vas » (Argentinien, über 38'000 Eintritte). 1995 lag noch ein weiterer argentinischer Film unter den grössten Kinoerfolgen, nämlich «Sur» (mehr als 60'000 Besucher 1995). Das kubanische Werk «Fresa y chocolate» schaffte es zwei Jahre hintereinander auf die Liste der 100 erfolgreichsten Filme (über 90'000 Besucher 1994 und 47’000 1995).

\section{$\square$ Förderung von Filmen aus Entwicklungsländern}

Seit Beginn der 80er Jahre werden die Produktion und die Verbreitung von Filmen aus anderen Kontinenten auf verschiedene Weise gefördert. Am Freiburger Filmfestival, das Anfang der 80er Jahre ins Leben gerufen worden war, wurden im März 1997 über 70 Produktionen vorgeführt. Aber auch an anderen Filmfestivals und -zyklen werden den Besuchern Bilder aus anderen Teilen der Erde geboten, so z.B. am Festival Cinemafrica, das alle zwei Jahre im November in 
Zürich stattfindet, sowie am Filmfestival von Locarno. An den von den kirchlichen Hilfswerken organisierten Film- und Videotagen Nord-Süd wird jeweils eine Auswahl von Filmen präsentiert, die von den Schulen ausgeliehen werden können ${ }^{9}$ (Filme für EINE Welt). Die in Genf stattfindenden «Medienbegegnungen Nord-Süd» führen jedes Jahr im Mai Dokumentarfilme von Fernsehanstalten und unabhängigen Produzenten vor. Die Stiftung Trigon-film verleiht ausschliesslich Filme von Regisseuren aus Afrika, Asien und Lateinamerika. Auf diese Weise konnten in den vergangenen zehn Jahren über 60 Filme der Öffentlichkeit zugänglich gemacht werden.

Die Direktion für Entwicklung und Zusammenarbeit (DEZA) ist ebenfalls in der Förderung des künstlerischen Schaffens und des kulturellen Austauschs aktiv. Sie unterstützt die Produktion und die Verbreitung von Filmen aus den südlichen Ländern und aus Osteuropa. Die DEZA erachtet das Kino als Sensibilisierungsund Informationsinstrument, die zum besseren Verständnis des «anderen» beiträgt, den Zugang zur Realität in anderen Ländern erleichtert und die Solidarität fördert. Das Kino stellt aber auch eine Möglichkeit für den Süden dar, etwas an die Gesellschaft des Nordens weiterzugeben und sich nicht nur als ewiger Hilfeempfänger zu präsentieren ${ }^{10}$. Die von der DEZA und Benetton Schweiz gemeinsam finanzierte Stiftung Montecinemaverità konnte seit Oktober 1992 die Produktion von 34 Filmen finanziell unterstützen.

Der Beitrag der DEZA an das Filmschaffen in den südlichen und osteuropäischen Ländern belief sich 1997 auf 830'000 Franken. Davon entfielen 200'000 Franken auf die Verbreitung von Filmen aus dem Süden in der Schweiz (Trigonfilm), 200'000 Franken auf die Organisation von Filmfestivals und -zyklen und 330'000 Franken auf die Beiträge an die Stiftung Montecinemaverità (die von Benetton Schweiz mit weiteren 310'000 Franken unterstützt wurde). Zu den Filmen, die in jüngster Vergangenheit von der Stiftung gefördert wurden, gehören insbesondere «Bab El Oued City ( Algerien), «L'arche du désert » des Algeriers Mohamed Chouikh, und «Dance of the Wind» (Indien). Neben der Trigon-film werden auch das Filmfestival von Freiburg, Cinemafrica, Black Movie, die Medienbegegnungen Nord-Süd sowie die Weltfilmtage von Thusis von der DEZA finanziell unterstützt. Überdies wurde 1997 ein weiterer Betrag von 100'000 Franken für die Finanzierung von Dokumentarfilmen zur Verfügung gestellt (1998 soll dieses Budget auf 125'000 Franken aufgestockt werden). Zusätzlich zu den 830'000 Franken zugunsten der Förderung des Filmschaffens stellte die DEZA 680'000 Franken für die Unterstützung des kulturellen Austauschs zur Verfügung (Musik, Theater, Publikationen), wovon ein Teil dem Programm « Kultur und Entwicklung» zugute kam.

9. Spiel- und Dokumentarfilme aus Entwicklungsländern, die Schulen (oder in gewissen Fällen Kinoklubs) zur Verfügung gestellt werden, sind an folgenden Adressen erhältlich:

Angebot der kirchlichen Hilfswerke: Zoom Filmverleih

Trigon-Filme (und andere): FilmInstitut, Tel. 031/301 0116 (Videos können bei der DEZA erworben werden).

Cinédia, C.P. 197, 1701 Fribourg, Tel. 026/426 3430 (Cinédia stellt eine Auswahl längerer und kürzerer Filme für nichtkommerzielle Vorführungen zur Verfügung und bietet Videos zum Kauf an).

10. Interview mit Marco Cameroni vom Informationsdienst der DEZA in La Regione Ticino, 14. August 1997. 
Rund 30 schweizerische Organisationen sind in unterschiedlichem Masse in der Förderung der Kunst in den Entwicklungsländern tätig (Musik, Filme, Theater, Literatur, Photographie, Ausstellungen, zeitgenössische Kunst, Promotion von Tourneen, Film- oder Musikfestivals). Mehrere nichtstaatliche Organisationen verfolgen unter anderem das Ziel, das Verständnis für andere Kulturen zu fördern". Mit ihrem Programm «Kulturaustausch Süd-Nord», welches über ein jährliches Budget von rund 430'000 Franken verfügt, möchte die Stiftung Pro Helvetia kulturelle Aktivitäten unterstützen, die einen Beitrag zum Dialog zwischen der Schweiz und den Entwicklungsländern leisten können. «Kultur und Entwicklung» ist ein von den Hilfswerken gemeinsam getragenes Programm, welches die Präsentation des kulturellen Schaffens aus anderen Kontinenten in der Schweiz unterstützt und koordiniert.

QUELLEN

Procinéma, Schweizerischer Verband für Kino und Filmverleih, Bern, 1997 (Statistiken 1996).

Basler Zeitung, 8./10. August 1997 (Stiftung Montecinemaverità).

La Regione Ticino, 14. August 1997 «Marco Cameroni illustra il ruolo della DSC all'interno di Montecinemaverità ».

11. Unter anderem sind folgende Institutionen in der Kulturförderung aktiv: Fastenopfer, Caritas, Erklärung von Bern, HEKS, Helvetas, Flüchtlingshilfeorganisationen, Brot für alle, Swissaid, Terre des hommes Schweiz und Unicef. 